\title{
Detection Of Biogenic Amines By The Use Of Advanced Chemometrics Tools
}

\author{
Marta Bonet-San-Emeterio, Xavier Cetó, Manel del Valle*
}

Sensors and Biosensors Group, Universitat Autònoma de Barcelona, Edifici CN, 08193

Bellaterra, Barcelona, Spain

Keywords: Biogenic Amines; Electronic tongue; Sensor array; Artificial Neural Networks; Electrochemistry; Cyclic Voltammetry; Histamine; Tyramine; Cadaverine

The present work proposes an electronic tongue arrangement for the detection of biogenic amines (BAs) in ternary complex mixtures.

Since, the formation of BAs is directly proportional to the increase of temperature and the presence of bacteria, the elevated concentration of that kind of compounds could be related easily with the quality of the food industry products. The most regulated field is the fish industry, who has set limits for histamine as the marker compound: $100 \mathrm{mg} \cdot \mathrm{Kg}^{-1}$ in the European Union and 50 $\mathrm{mg} \cdot \mathrm{Kg}^{-1}$ in the United States of America.

Herein it is proposed a voltammetric sensor array for the quick and efficient detection of histamine (Hys), cadaverine (Cad) and tyramine (Tyr). Which together with advanced chemometric tools such as artificial neural networks (ANN) and partial least squares (PCA), lead to models able to predict the individual concentration of each BA in the analyzed samples.

The final ANN structure had 51 input neurons, 5 neurons in the hidden layer and 3 neurons in the output layer. The functions used for the hidden and output layers were Tansig and Purelin, respectively. The results show that this is a valid model with slopes near to 1 and intercepts close to 0 . Moreover, it is important to remark that the worst correlation has a value of almost 0.900 . 